\title{
ASSOCIATIONS OF SONG PROPERTIES WITH HABITATS FOR TERRITORIAL OSCINE BIRDS OF EASTERN NORTH AMERICA
}

\author{
R. Haven Wiley \\ Department of Biology, University of North Carolina, Chapel Hill, North Carolina 27599-3280
}

Submitted January 26. 1990; Revised August 8, 1990; Accepted September 19, 1990

\begin{abstract}
To investigate adaptations for long-range acoustic communication in birds, I analyzed associations between broad categories of habitats and properties of territorial songs for eastern North American oscines. From published recordings, I obtained three frequency properties (maximal, minimal, and dominant) and three temporal properties of songs (presence of sidebands, presence of buzzes, minimal period of repeated elements). Sidebands and buzzes indicated rapid amplitude modulation of a carrier frequency. Habitats occupied by territorial males were classified into six categories (broad-leaved or mixed forest, coniferous forest, parkland or forest edge, shrubland, grassland, and marshes). Frequencies in songs correlated strongly with body size, which varied among habitats. Analysis of covariance and phylogenetic regression, after controlling for body size, revealed an association of maximal but not dominant or minimal frequencies with habitat. In contrast, the temporal properties of song were all strongly associated with habitat, even within phylogenetic groupings. These results suggest that the temporal properties of songs of many oscines have evolved to reduce the effects of reverberation in forested habitats. Exceptional species might have retained features of song subject to degradation to permit listeners to judge distances to singers. In addition, adaptations for acoustic communication in different habitats might include differences in the perception of songs.
\end{abstract}

Attempts to discover adaptations in the structure of birds' songs to particular habitats have taken two directions: surveys of the characteristics of songs and habitats of a wide variety of species in a geographical area (Chappuis 1971; Morton 1975; Richards and Wiley 1980; Ryan and Brenowitz 1985; Sorjonen 1986a) and detailed analyses of song structure in relation to sound propagation in sets of a few populations or related species (Jilka and Leisler 1974; Nottebohm 1975; Bowman 1979; Hunter and Krebs 1979; Wasserman 1979; Gish and Morton 1981; Handford 1981; Lemon et al. 1981; Shy 1983; Cosens and Falls 1984; Anderson and Conner 1985; Sorjonen 1986b). The first has advantages in determining broad trends in adaptations of acoustic signals, the latter, in more detailed understanding of the physical basis for these adaptations. This study takes the first course in surveying nearly all the territorial oscines of eastern North America to investigate the relationships between acoustic features of songs and broad categories of habitats.

Sound propagating through natural environments is subject to overall attenuation by spherical spreading and, in addition, to frequency-dependent attenuation, reverberation, and irregular amplitude fluctuation (Wiley and Richards 1978, 1982). The term degradation, as used here, refers to the combined effects of 
reverberation and amplitude fluctuation, as opposed to frequency-dependent attenuation (see Wiley and Richards 1982). This study investigated two specific predictions for adaptations in the structure of long-range acoustic signals that could minimize the effects of reverberation, on the one hand, and frequencydependent attenuation, on the other.

Greater reverberation in forests as opposed to open habitats (Richards and Wiley 1980; Wiley and Richards 1982) leads to predictions that forest-inhabiting species should avoid features easily degraded by reverberation. In a survey of passerines breeding in North Carolina, Richards and Wiley (1980) found evidence that many forest-inhabiting species had territorial songs that avoided short repetition periods between elements at the same frequency. Other studies have reported similar differences (Nottebohm 1975; Handford 1981; Anderson and Conner 1985; Sorjonen $1986 a, 1986 b$ ), but no study so far has explicitly investigated this prediction in a large sample of species.

Patterns of frequency-dependent attenuation in forests and open habitats suggest that forest-inhabiting species should use lower frequencies (Chappuis 1971; Morton 1975). The rationale for this prediction is not so clear as for the previous one since, in all habitats and for all frequencies within the range of those used by oscines, lower frequencies should propagate better than higher ones, except within a meter or so of the ground (Marten and Marler 1977; Marten et al. 1977; Wiley and Richards 1982; Waser and Brown 1984). Several comparisons of related species or populations have provided some evidence for adaptations in the frequencies in song (Jilka and Leisler 1974; Bowman 1979; Hunter and Krebs 1979; Wasserman 1979; Lemon et al. 1981; Shy 1983; Cosens and Falls 1984; Anderson and Conner 1985). Two surveys of a Neotropical avifauna have also confirmed associations of frequencies in song with broad categories of habitat (Morton 1975; Ryan and Brenowitz 1985). No such analysis is available for a diverse temperate avifauna.

In analyzing associations of habitat and features of song, it is important to consider two possible confounding variables. First, the dimensions of a sound source affect the frequencies that are produced most efficiently (Kinsler and Frey 1962). For this reason, of course, the frequencies in birds' songs are strongly related to body size (Wallschläger 1980; Ryan and Brenowitz 1985). Second, phylogenetic trends might result in groups of species sharing particular characteristics, such as features of songs and habitats. Ryan and Brenowitz (1985) report such effects in comparisons among oscine, suboscine, and nonpasseriform birds. Thus, this study, in examining possible associations of the temporal features and frequencies in songs with broad categories of habitat, also considered the confounding effects of body size and phylogenetic relationships.

\section{METHODS}

\section{Species Included}

This study included the songs of 120 species of oscines in the Field Guide to Bird Songs (Cornell Laboratory of Ornithology 1975). I excluded a few species 
that do not use song for advertisement of relatively large territories (all swallows and corvids; golden-crowned kinglet, Regulus satrapa; cedar waxwing, Bombycilla cedrorum; common grackle, Quiscalus quiscula; brown-headed cowbird, Molothrus ater; pine siskin, Carduelis pinus; evening grosbeak, Coccothraustes vespertinus). This sample thus included nearly all territorial oscines breeding regularly between the Rocky Mountains and the Atlantic coast of North America. For some analyses, species were classified by subfamilies and genera as recognized by the American Ornithologists' Union (1983).

These recordings include a representative sample of songs for each species (usually 1-3 distinct song patterns; maximum, 8). There is no reason to think that they were selected with the hypotheses in mind. Although some of the recordings clearly were subjected to high- or band-pass filtering to eliminate background noise, none of the spectral analyses showed indications that this filtering had encroached on the frequencies in the songs. Any such filtering would make the measurements of extreme frequencies in songs less reliable.

For each species, I obtained the mean body mass of adult males from Dunning (1984), except for Bachman's warbler Vermivora bachmanii, and chipping and field sparrows Spizella passerina and Spizella pusilla (not included in this reference). For the latter two species, I used lean masses obtained by Murray and Jehl (1964). Sound sources radiate energy most efficiently at frequencies near the resonant frequency of the source. This frequency generally varies inversely with characteristic linear dimensions of the resonator (Kinsler and Frey 1962, p. 193). Assuming that such considerations apply to birds, I have used the inverse cube root of body mass as a measure of overall size in the following analyses.

\section{Classification of Habitats}

The usual habitats occupied by territorial males of these species were classified on the basis of the height and density of the vegetation and the locations of usual singing posts in relation to the top of the vegetation: (1) broad-leaved or mixed forest with trees forming a closed canopy over areas as large as or larger than territory sizes; (2) parkland with trees either not contiguous or in clusters smaller than territory sizes (including edges of forests); (3) shrubland with woody vegetation generally low in stature, including habitats with patches of shrubs (hedgerows and brushy fields); and (4) grasslands with woody vegetation sparse or absent. In categories (1) and (2) birds rarely sang from positions above the highest vegetation, whereas in categories (3) and (4) birds regularly sang from the tops or above the top of the highest vegetation. In addition, two more narrowly defined habitats were recognized: (5) predominantly coniferous closed forest and (6) herbaceous marshes with emergent vegetation in standing water. In some analyses, these six categories were lumped into two: forest and parkland (1,2, and 5) as opposed to open habitats $(3,4$, and 6$)$. In classifying each species' predominant habitat, I consulted references that included relatively systematic descriptions of breeding habitats over entire states or provinces (Stewart and Robbins 1958; Imhof 1962; Stewart 1975; Salt and Salt 1976; Laughlin and Kibbe 1985).

The habitats occupied by breeding birds clearly vary among individuals within a population and among populations of the same species. Individual birds in 
some cases even incorporate two or more of the above habitats in their breeding territories. Thus, an attempt to classify each species by its predominant breeding habitat can only approximate a classification of the actual conditions in which many members of that species actually sing. Furthermore, the habitat occupied by each individual on the recordings used in this analysis is not known. The lack of a match between the actual habitats of the subjects and the habitats selected for each species in this study other than that from unsuspected systematic error would reduce the chances for detecting significant associations of song structure and habitat. These considerations make the statistical results of this analyses conservative.

\section{Measurements of Songs}

Sound spectrograms of songs were prepared by the method described by Hopkins et al. (1974). Briefly, the output of a Spectral Dynamics 512-line real-time spectrum analyzer was filmed continuously to produce a sound spectrogram on $35-\mathrm{mm}$ photographic paper. For these analyses I used an analysis range of 50 $\mathrm{kHz}$ with an analysis period of $50 \mathrm{~ms}$ but limited the display to $20 \%$ of the range to produce a spectrogram with a frequency resolution of $100 \mathrm{~Hz}$ and a temporal resolution of $10 \mathrm{~ms}$ (confirmed by examination of the frequency-axis ramp by oscilloscope).

Frequencies on these spectrograms were measured by ruler with a precision of $300 \mathrm{~Hz}(0.5 \mathrm{~mm})$ and scaled by measurement of known frequencies. Temporal measurements were also made by ruler with a precision of $10 \mathrm{~ms}$.

To determine the dominant frequencies in songs, I averaged spectra over periods of $1.5 \mathrm{~s}$ by transferring the 256 spectra from one frame (slightly more than the amount displayed on the screen) of a Uniscan II spectrum analyzer to a microcomputer. The dominant frequency in these averaged spectra was taken as the frequency with the highest spectral amplitude (rounded to the nearest $100 \mathrm{~Hz}$ ).

For each species in the sample, I took the following measures of its songs: (1) maximal frequency across all songs; (2) minimal frequency across all songs; (3) mean dominant frequency (average of the dominant frequencies in each distinct song type or, if songs were longer than $1.5 \mathrm{~s}$, in each 1.5 -s portion of all songs); (4) minimal period of repeated units (such as syllables in a trill; omitted in 15 species that lacked repeated units); (5) presence of harmonics; (6) presence of sidebands; and (7) presence of one or more buzzes (notes with a wide but bandlimited spectrum).

Harmonics are evenly spaced frequency components that represent integer multiples of a fundamental, which in some cases is absent or attenuated. Sidebands are the representation in the frequency domain of amplitude or frequency modulation at rates greater than the reciprocal of the spectral analysis period. Usually they appear as closely spaced frequency components on either side of a more prominent central frequency, an indication of rapid amplitude modulation of the central frequency (see illustrations in Stein 1968; Marler 1969). Buzzes often intergrade with very closely spaced sidebands. Some experimentation often reveals that they represent sidebands close to the frequency resolution of the analysis (or, equivalently, amplitude modulation at rates close to the temporal 
TABLE 1

Correlations and Associations of Variables Included in This Study

\begin{tabular}{lcccccc}
\hline & BZ & RP & MX & MN & DM & MS \\
\hline SB & $33.6^{* *}$ & 1.13 & -.09 & -.05 & -.02 & .17 \\
BZ & & $9.89^{* *}$ & .08 & -.08 & .01 & .17 \\
RP & & & -.08 & .08 & .03 & .01 \\
MX & & & $.41^{* *}$ & $.57^{* *}$ & $-.36^{* *}$ \\
MN & & & & $.86^{* *}$ & $-.35^{* *}$ \\
DM & & & & & $-.30^{* *}$ \\
\hline
\end{tabular}

NotE.-SB, sidebands; BZ, buzzes; RP, minimal repetition periods $<10 \mathrm{~ms}$; MX, maximal frequency; MN, minimal frequency; DM, dominant frequency; MS, body mass. The three entries for the associations between dichotomous variables $(\mathrm{SB}, \mathrm{BZ}$, and $\mathrm{RP})$ are values of $\chi^{2}(\mathrm{df}=1)$; all other entries are Pearson correlation coefficients $(N=119)$.

** $P<.01$.

resolution of the analysis). Sidebands, buzzes, and short repetition periods thus represent amplitude modulation with progressively longer periods.

\section{Statistical Analysis}

A program for generalized linear modeling (GLIM, Royal Statistical Society, London; Healy 1988) was used to evaluate the influences of body mass, phylogenetic groupings, and habitat on each measure of the species' songs. The measures of frequencies in songs (dominant, maximal, and minimal) correlated with each other; the measures of temporal structure (sidebands, buzzes, minimal repetition periods) also showed strong associations (table 1). Rather than extract principal components of these measures, I tested each separately for associations with the predictor variables because the separate measures permit clearer biological and physical interpretation.

For measures of frequencies, ANCOVAs examined the differences among habitats, with the inverse cube root of body mass as a covariate. A significant interaction of habitat and mass in these analyses would indicate that the slope of the regression of frequency on mass differed among habitats. A significant effect of habitat would indicate that the regressions of frequency on inverse cube root of mass differed in elevation among habitats.

For measures of temporal properties of songs, variables that were either categorical or highly skewed, three-way contingency tables were analyzed by means of log-linear models. In these analyses each species counted as a single observation in a $6 \times 2 \times 2$ table, with the six categories of habitat described above, the measure of a temporal property of songs divided into two categories (if not already in two classes) at the median of the overall distribution, and a third variable also in two categories (either two phylogenetic categories or two categories of body mass again divided at the median). To evaluate the effect of the three-way interaction, I compared the full model to one with the three-way interaction eliminated. In every case the increase in deviance (also called $G^{2}$ ) fell far short of statistical significance, based on a $\chi^{2}$ approximation for differences between deviances in models with Poisson error distributions. The three-way interaction 
was thus eliminated from further consideration. Next, I considered three models, each excluding one of the three possible two-factor associations among the variables. For each of these models, the increase in deviance over that for the model with all possible two-factor associations provided a measure of the importance of the missing association. Statistical significance was judged by referring the difference in deviance to the $\chi^{2}$ distribution for the appropriate degrees of freedom (for further discussion, see McCullagh and Neder 1983). Since a $6 \times 2 \times 2$ classification resulted in some sparse or empty cells, I also analyzed these data in $2 \times 2 \times 2$ classifications with the same procedures, except that habitats were classified in only two categories, as described above.

These conventional analyses are not completely satisfactory for comparative studies. In testing for associations of characters in phylogenetically related species, it is not correct to assume that each species is a statistically independent observation. Two species might share similar traits as a result of having common ancestors with these traits and thus not represent independent instances of convergent evolution. In discussing this issue, Ridley (1983) proposed that each independently evolved instance of an association of characters should count only once.

This approach has been adapted by Grafen (1989) in a procedure for phylogenetic regression, which reduces each separate radiation in the dependent variable to a single point in a final ANCOVA. This program is used here to check the results from the conventional ANCOVAs for dominant, maximal, and minimal frequencies in songs, variables that are continuous with approximately normal distributions. For each variable I used the phylogenetic regression with the categories of habitats as test variables and the inverse cube root of mass as a control variable and again with these test and control variables interchanged.

For measures of the properties of songs that were either categorical (presence or absence of sidebands, buzzes, and harmonics) or highly skewed (minimal repetition intervals), the phylogenetic regression was not appropriate since the fitted values of these variables included many far outside the original distributions. Ridley's method of independent instances of convergent evolution would apply here, provided that ancestral states of these characters at each node in an acceptable phylogeny could be inferred, for instance by out-group analysis (Ridley 1983, 1989). However, the characters of song vary widely in subfamilies and genera included in this analysis (the data include only four subfamilies with two to four species each and five genera also with two to four species each that are uniform for the presence or absence of sidebands or buzzes). This situation, combined with uncertainties in the phylogeny of taxa of oscines, makes the inference of ancestral states for many genera and subfamilies highly problematic. Consequently, any count of independent instances of convergent evolution in characteristics of habitat and song is unreasonably speculative.

Instead, I adopted two modified procedures for dealing with the possible lack of independence among related species, one a mild solution, the other a radical one. The "mild solution" consisted of counting any taxa that were uniform for the measure of song (the dependent variable) as a single case. For the associated explanatory variable (two categories of habitat or mass), I took the most frequent 
value for species in that taxon. The "radical solution" (suggested by A. Grafen [personal communication]) involved focusing exclusively on those higher phylogenetic nodes (taxonomic categories above the level of species) for which all daughter nodes (next-lower taxonomic categories including species) were both separately uniform for the explanatory variable and different among themselves. For each such higher node, the null hypothesis was that there was no difference between values of the dependent variable among daughter nodes with different values of the explanatory variable, as judged by either a binomial test or a Wilcoxon matched-pairs signed-ranks test. In analyzing the associations of harmonics, sidebands, buzzes, and minimal repetition periods (divided at the median) with habitat, this sort of analysis excluded all species except those in 10 genera or subfamilies, for which details are presented below. Although the original data were thus radically pruned, these analyses are as nearly as possible beyond suspicion of inflated degrees of freedom as a result of associations of characters by common ancestry.

RESULTS

\section{Frequency Characteristics of Songs}

In the ANCOVAs for the dominant, maximal, and minimal frequencies in songs, measures of frequencies varied strongly and consistently with body mass (table 2; figs. 1 and 2). In the analysis with six categories of habitats, only maximal frequencies differed significantly among habitats (table $2 \mathrm{~A}$ ). With only two categories of habitat, on the other hand, only dominant frequencies varied between habitats, with marginal statistical significance (table 2B). Note that the habitat with the highest mean dominant frequency, coniferous forests (fig. 1), also had the smallest mean body mass (fig. 3). Conversely, both the lowest mean dominant frequency and the largest mean body mass occurred in marshes.

The acoustic properties of coniferous forest and herbaceous marsh might mask differences between other forest and open habitats. The small leaves of conifers result in less attenuation of frequencies between 2 and $8 \mathrm{kHz}$ than in broad-leaved forests (Marten and Marler 1977), so species might use higher frequencies in relation to their body size in coniferous than in other forests. On the other hand, there is less attenuation of low frequencies in marshes than in grasslands (Cosens and Falls 1984), so species might use lower frequencies in marshes than in other open habitats. If species inhabiting coniferous forests and marshes were excluded, an ANCOVA revealed differences among the remaining four habitats in all three measures of frequencies in songs (table $2 \mathrm{C}$ ). Maximal frequencies in particular showed highly significant differences among habitats. In an analysis with only grassland and marsh as categories of habitat, although the number of species was much lower, there was also a difference in maximal frequencies (table 2D).

Phylogenetic regression provided a check against the possibility that these results were influenced by inflated degrees of freedom as a result of phylogenetic associations of the variables. In analyses with six categories of habitats, when 
TABLE 2

Results of an ANCOVA of the Frequencies of Songs

in Different Habitats with the Inverse Cube Root of Body Mass as a Covariate

\begin{tabular}{|c|c|c|c|}
\hline \multirow[b]{2}{*}{ Frequency Measure } & \multicolumn{3}{|c|}{ SOURCES OF VARIANCE } \\
\hline & Habitat & Mass & Interaction \\
\hline \multicolumn{4}{|c|}{$\begin{array}{l}\text { A. Six categories of habitat } \\
(N=119) \text { : }\end{array}$} \\
\hline $\mathrm{df}$ & 5,107 & 1,107 & 5,107 \\
\hline Dominant frequency & 1.92 & $37.76^{* *}$ & 1.34 \\
\hline Maximal frequency & $3.69^{* *}$ & $24.66^{* *}$ & .62 \\
\hline Minimal frequency & .20 & $36.92 * *$ & 1.45 \\
\hline \multicolumn{4}{|c|}{$\begin{array}{l}\text { B. Two categories of habitat } \\
(N=119) \text { : }\end{array}$} \\
\hline $\mathrm{df}$ & 1,115 & 1,115 & 1,115 \\
\hline Dominant frequency & $4.54^{*}$ & $30.01^{* *}$ & .55 \\
\hline Maximal frequency & 1.96 & $24.66^{* *}$ & .29 \\
\hline Minimal frequency & 3.15 & $41.66^{* *}$ & .07 \\
\hline \multicolumn{4}{|c|}{$\begin{array}{l}\text { C. Four categories of habitat } \\
\text { (excluding species in coniferous } \\
\text { forest and marsh) }(N=103) \text { : }\end{array}$} \\
\hline $\mathrm{df}$ & 3,95 & 1,95 & 3,95 \\
\hline Dominant frequency & $3.10^{*}$ & $34.16^{* *}$ & 1.15 \\
\hline Maximal frequency & $5.62^{* *}$ & $21.55^{* *}$ & .14 \\
\hline Minimal frequency & $2.76^{*}$ & $43.15^{* *}$ & .76 \\
\hline \multicolumn{4}{|c|}{$\begin{array}{l}\text { D. Habitat categories of grassland } \\
\text { and marsh only }(N=26) \text { : }\end{array}$} \\
\hline df & 1,22 & 1,22 & 1,22 \\
\hline Dominant frequency & 1.77 & $5.64^{*}$ & 1.44 \\
\hline Maximal frequency & $5.57 *$ & $14.70^{* *}$ & .34 \\
\hline Minimal frequency & 3.38 & $9.68^{* *}$ & .80 \\
\hline
\end{tabular}

NotE.-Entries are the $F$-ratios for each variable or interaction, with the specified degrees of freedom.

$* P<.05$.

$* * P<.01$.

habitat was controlled, dominant frequency was strongly related to body size (table 3A). The results were similar but less marked for the measures of maximal and minimal frequency. On the other hand, when body size was controlled, only maximal frequency differed among habitats, with marginal statistical significance (table 3B). If coniferous forests and marshes were omitted from the analysis, the results were very similar, although the $F$-values were larger for effects of both size and habitat on measures of frequencies (table 3C). In this case, the differences in maximal frequencies among habitats were highly significant.

These analyses thus provided little evidence for associations of the general characteristics of frequencies in songs with gross features of vegetation, provided the influence of body size was controlled. Both conventional ANCOVAs and phylogenetic regression indicated that only maximal frequencies differed consistently among habitats. On the other hand, all measures of frequencies in birds' songs depended strongly on body size. 
Dominant $\square$ Maximal $\square$ Minimal

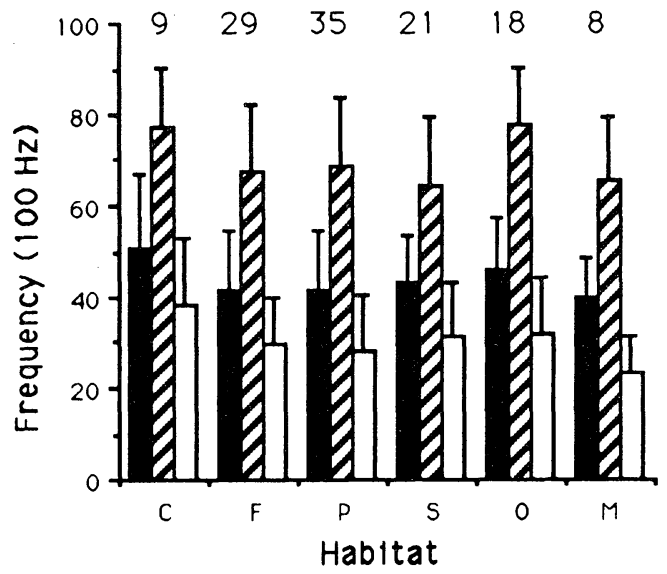

FIG. 1.-Means and standard deviations of dominant, maximal, and minimal frequencies in songs of oscines in six broad categories of habitat: $F$, broad-leaved or mixed forest; $P$, parkland or forest edge; $S$, shrubland; $O$, open grassland; $C$, coniferous forests; and $M$, marshes. Numbers above the columns indicate the species in each habitat.
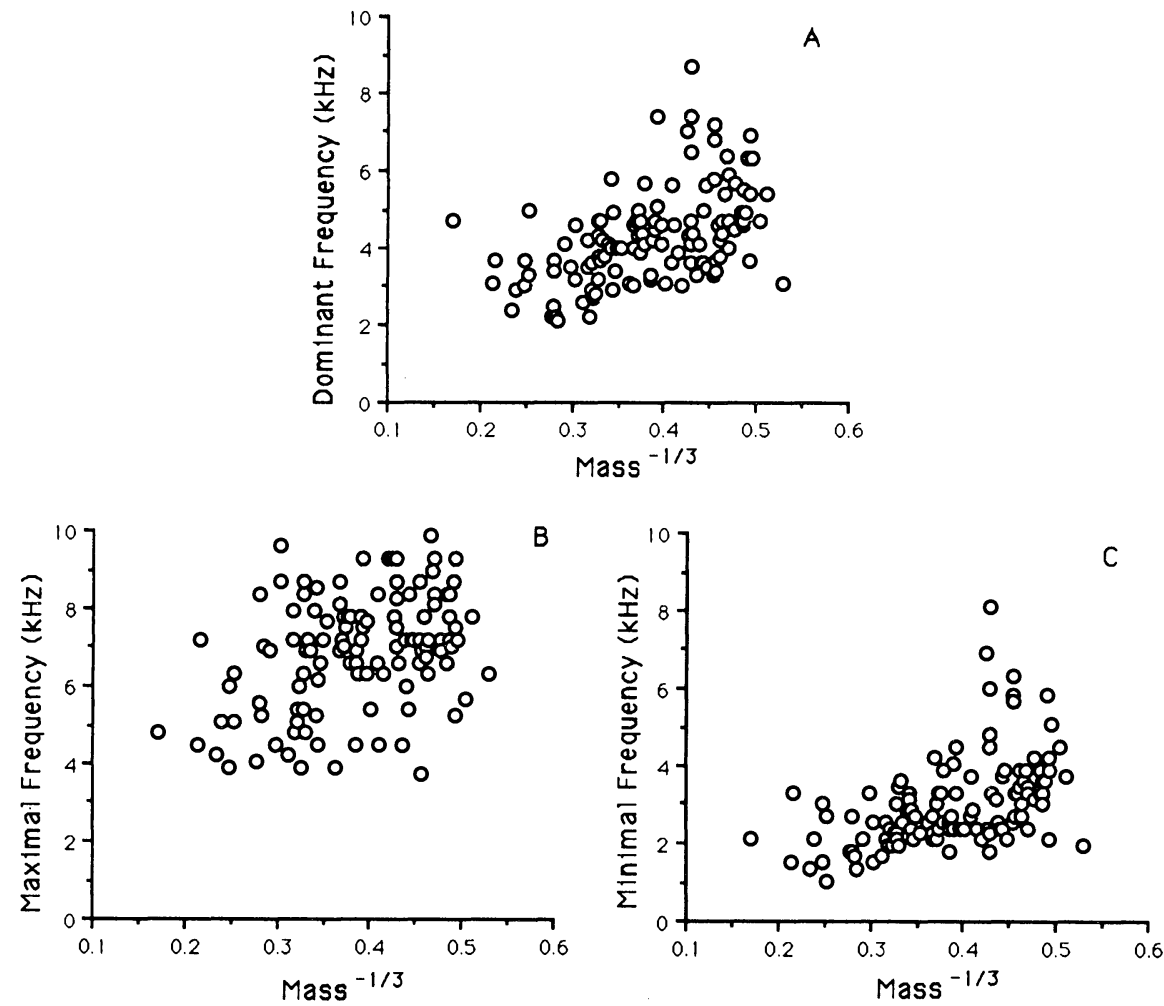

FIG. 2.-Dominant, maximal, and minimal frequencies in 119 species' songs as a function of the inverse cube root of body mass $(\mathrm{g})$. 


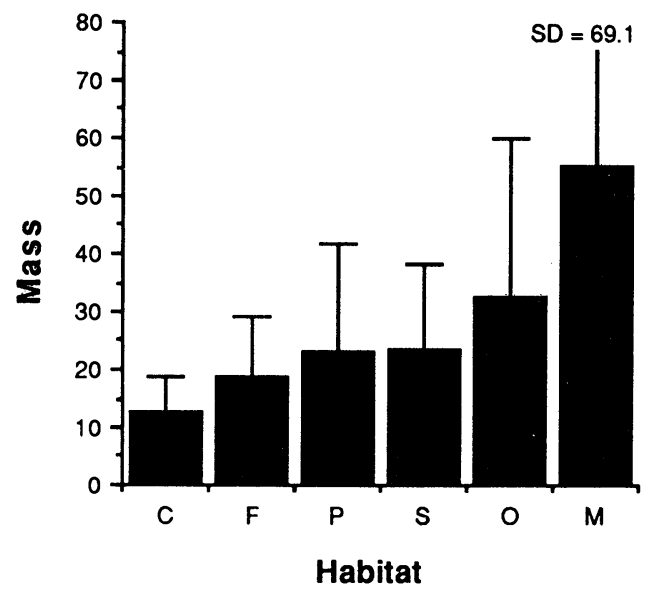

FIG. 3.-Body mass (g) of oscines in six broad categories of habitats. Numbers of species in each habitat are as in fig. 1.

TABLE 3

Results of the Phylogenetic Regression of Frequency Characteristics of Song on the Inverse Cube Root of Body Mass While Controlling the Effects of Habitat and Vice Versa

Frequency Measure
A. Regression of frequency char-
acteristics on the inverse
cube root of body mass, con-
trolling for habitat (six cate-
gories):

Dominant frequency

Maximal frequency

Minimal frequency

.202

.111

.302

1,24

1,25

1,24

$9.77 * *$

$7.55^{*}$

$5.27^{*}$

B. Regression of frequency characteristics on habitat ( $\mathrm{six}$ categories), controlling for inverse cube root of body mass:

Dominant frequency

Maximal frequency

.149

.082

Minimal frequency

C. Regression of frequency characteristics on habitat (four categories, excluding coniferous forest and marsh), controlling for inverse cube root of body mass:

Dominant frequency

Maximal frequency

Minimal frequency
5,25

5, 25

5, 25 $\mathrm{df}^{\mathrm{b}}$

F

${ }^{a} \rho$ is the exponent of the power function used in weighting the hierarchy of phylogenetic nodes in the final model; it provides an indication of the importance of the phylogeny in explaining the associations of the dependent and test variables (see Grafen 1989).

${ }^{b}$ df can vary for the same set of species depending on whether one or more nodes lack "phylogenetic degrees of freedom" for a particular variable (Grafen 1989).

$* P<.05$.

** $P<.01$. 


\section{ain. Repetition Period $>10 \mathrm{~ms}$ \\ $\square$ Side Bands Absent}

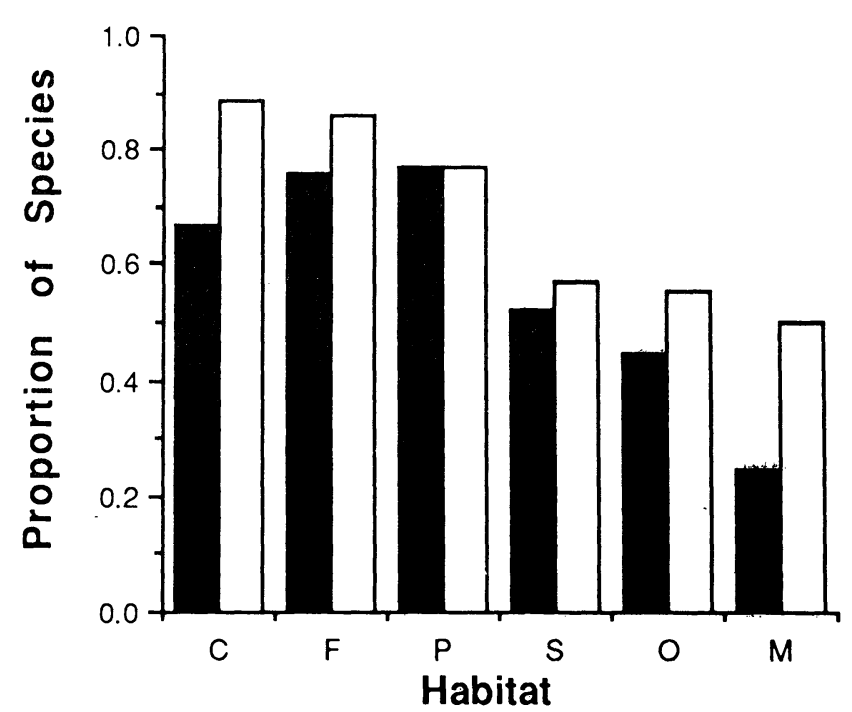

FIG. 4.-Proportions of oscines in each broad category of habitat that lack sidebands in their songs and that have minimal repetition periods greater than $10 \mathrm{~ms}$. Numbers of species in each habitat are as in fig. 1.

Contingency tables revealed no significant associations between the frequency characteristics of songs and phylogenetic categories within classes of body size.

\section{Harmonics and Temporal Characteristics of Song}

In open habitats, especially grasslands, most species included very short repetition periods in their songs and nearly half included notes with sidebands (fig. 4). In contrast, most forest-inhabiting birds included no short repetition periods in their songs, and only a small fraction included notes with sidebands. The presence of buzzes showed very similar patterns.

These temporal features of songs seemed likely, on first inspection, to have significant associations with phylogenetic groupings. For instance, the two largest subfamilies of oscines in eastern North America, the Parulinae and the Emberizinae, are not equally distributed across the broad categories of habitat used here. Parulinae are primarily forest and parkland species, whereas Emberizinae are primarily species of shrub and open habitats. The former are known for their melodic tonal songs, the latter for their trills and buzzes.

To focus on this potentially confounding influence on the temporal structure of songs, I analyzed just the 66 species of Parulinae and Emberizinae in a $2 \times 2 \times$ 2 classification of habitat, song feature (two categories divided at the median), and subfamily (two categories). This analysis did not consider harmonics since only one species in each subfamily included harmonics in its songs. The results 
TABLE 4

Changes in Deviance associated with Two-Factor Associations in Three-Way Contingency Tables for Temporal Features of Songs $(T)$, Habitats $(H)$, and Subfamilies $(S)$

\begin{tabular}{lccc}
\hline & \multicolumn{3}{c}{ Two-FActor Associations } \\
\cline { 2 - 4 } Song Feature & $T \times H$ & $T \times S$ & $H \times S$ \\
\hline A. $2 \times 2 \times 2$ table: $^{\text {a }}$ & & & \\
df & 1 & 1 & 1 \\
Sidebands & $9.09^{* *}$ & .47 & $28.19^{* *}$ \\
Buzzes & $7.82^{* *}$ & .95 & $19.16^{* *}$ \\
Minimal repetition period & $8.05^{* *}$ & .01 & $23.02^{* *}$ \\
B. $2 \times 2 \times 3$ table: & & 2 & 2 \\
df & 1 & $15.33^{* *}$ & $32.02^{* *}$ \\
Harmonics & 1.64 & 3.40 & $29.51^{* *}$ \\
Sidebands & $8.29^{* *}$ & 1.29 & $19.06^{* *}$ \\
Buzzes & $17.83^{* *}$ & 1.08 & $23.58^{* *}$ \\
Minimal repetition period & $7.20^{* *}$ & & \\
\hline
\end{tabular}

NotE.-Changes in deviance are the increase over the corresponding model including all two-factor associations but excluding the three-factor association (see Methods). In each case, eliminating the three-factor association had little effect on deviances.

${ }^{a}$ Parameters are two categories of song features (either presence/absence or divided at the median), two categories of habitats, and two subfamilies (Parulinae and Emberizinae).

${ }^{\mathrm{b}}$ Parameters are as above but with three subfamilies (Parulinae, Emberizinae, and all others).

$* * P<.01$.

clearly indicated pronounced associations of habitat with the presence of sidebands and buzzes and with minimal repetition periods, but no significant associations of song features with subfamily (table 4A; fig. 5). As expected, these analyses confirmed strong differences in the distribution of these two subfamilies between habitats. Variations of this procedure (using four categories of habitat, by lumping categories 1 with 5 and 4 with 6 ; excluding species in marshes; using three phylogenetic categories, Parulinae, Emberizinae, and all others) all yielded similar results. There was a strong association of buzzes with habitat in an analysis with three phylogenetic groups (table 4B). In these analyses there was no association of harmonics with habitat. Only 16 species included harmonics in their songs, 8 in each of two major categories of habitat (habitats 1, 2, and 5 and habitats 3,4 , and 6). Unlike the measures of temporal properties, harmonics had a strong association with subfamilies (table 4B).

The two corrections for phylogenetic associations of traits, as described in the section on methods, confirmed these results. The "mild solution" of counting only once each taxon that was uniform for the feature of songs under investigation indicated strong associations of the temporal features of songs, but not harmonics, with habitats. The "radical solution" of focusing only on mother nodes with uniform daughter nodes, within the phylogeny based on taxonomy, limited the analysis to 10 taxa: three subfamilies (Troglodytidae, Icterinae, Carduelinae) and seven genera (Hylocichla, Vermivora, Dendroica, Oporornis, Passerina, Spizella, Zonotrichia). The results, nevertheless, once again indicated strong ef- 


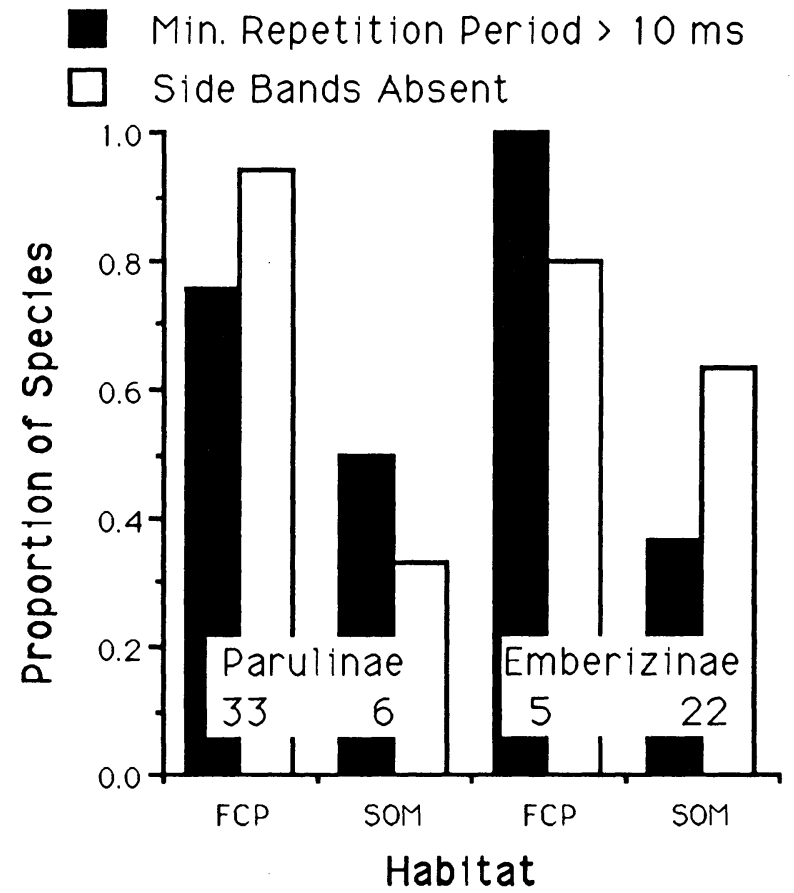

FIG. 5.-Comparison of Parulinae and Emberizinae for temporal characteristics of songs in two broad categories of habitats: $F C P$, forested habitats (including coniferous forest and parkland); $S O M$, open habitats (including shrubland and marsh). Numbers within each set of columns indicate the species in each subfamily and category of habitat.

fects of habitat (divided into two categories) on the presence of sidebands, buzzes, and minimal repetition periods (table 5).

In three-way contingency tables for temporal characteristics of songs, categories of body size, and habitats, there were no indications of associations between temporal features of song and body size within habitats.

\section{DISCUSSION}

These analyses demonstrate that habitat has a pronounced effect on the structure of the songs of oscine birds in eastern North America, but the effect is greater on the temporal structure of songs than on their frequencies.

For the frequencies in songs, a confounding variable was body size, since sources with smaller dimensions are more efficient at producing higher frequencies (Kinsler and Frey 1962). Although the syrinx and associated sound-producing structures of oscines probably do not have a fixed relationship with overall size, they are presumably correlated with a measure of overall size such as body mass. In the present analyses an association of frequency characteristics with body 
TABLE 5

Results of the "Radical Solution" to Controlling Phylogenetic Associations of Song FEATURES WITH HABITAT (FOR FEATURES WITH CATEgORICAL OR HIGHLY SKEWED DISTRIBUTIONS)

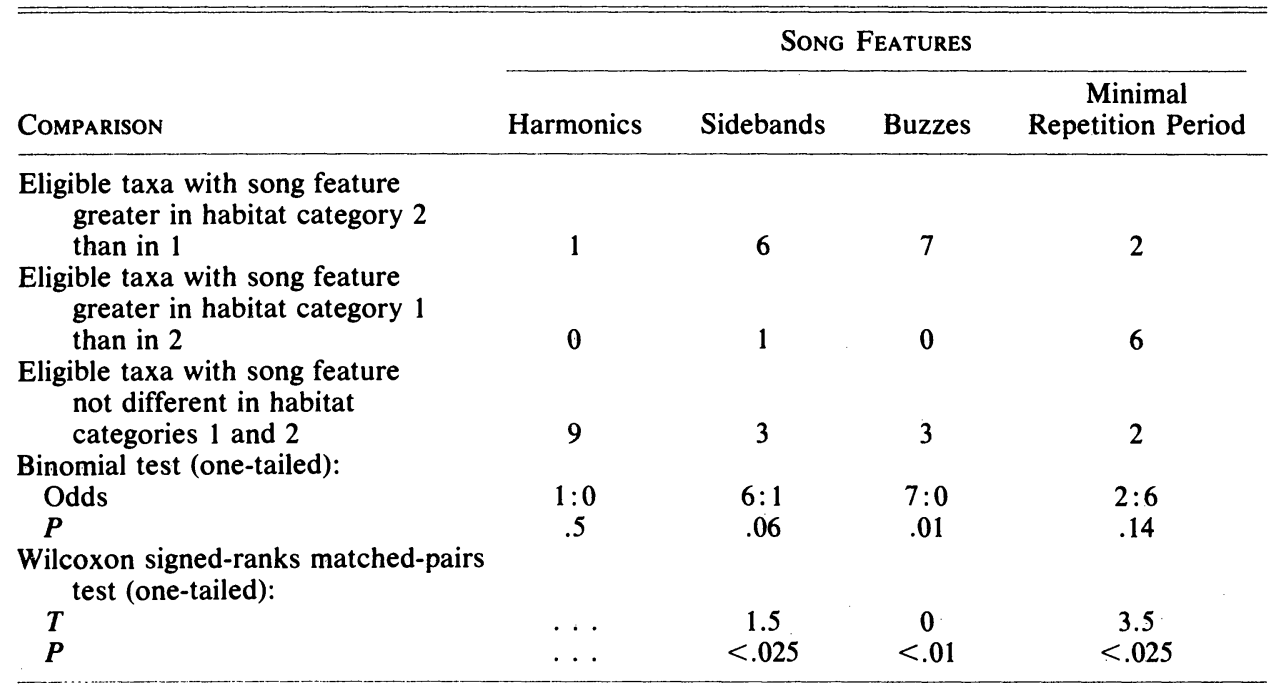

Note.-Eligible taxa are those mother nodes in the phylogeny based on taxonomy that have daughter nodes that are uniform for the feature of song in question (see the text); habitat category 1 includes forest (including coniferous forest) and parkland; habitat category 2 includes shrubland, grassland, and marsh.

mass appeared clearly. The association of body size with habitats in turn explained almost all of the variation among habitats in the frequencies in songs.

The temporal features of song on the other hand showed no relationship with body size. These measures of song structure instead seemed more plausibly associated with phylogenetic groups. Selection of the Parulinae and Emberizinae for detailed analysis focused attention on two sets of species, approximately equally numerous, which represented slightly over half of all species in the sample. Although the temporal structure of song in these two subfamilies differed strikingly, analysis showed that most of this variation was associated with differences in the habitats occupied by most of the species in each group. In fact, there was no indication that phylogenetic constraints influenced the temporal properties of song in these two subfamilies at all.

Harmonics are generated by nonlinearities in the sound-producing mechanism. Harmonics should be held to a minimum in all long-range advertising signals in order to concentrate maximal energy at any moment into a single frequency, which would increase the range of detection for receivers with frequencyanalyzing ears, like listening birds (Wiley and Richards 1982). There is no reason to expect the presence of harmonics to vary with habitat. The present results have in fact shown that harmonics occur infrequently in the advertising songs of oscines and have no association with habitats. 
These analyses represented no more than a coarse examination of the possible associations between habitat and song structure. The many statistical tests performed on the same data and the correlations among the independent variables suggest that an appropriate level for statistical significance should be lower than usual. The nature of the data itself provides additional reasons for the results not to be interpreted too closely: only a few songs of each species were measured; these exemplars were recorded in unknown habitats and circumstances; habitats were classified into only a few broad categories; and each species was assigned to a single predominant habitat. In addition, although I excluded species that use songs exclusively for communication at ranges of only a few meters, the species included in the sample vary widely in the usual distances between singers and potential receivers. An analysis of this sort inevitably misses any fine-scaled associations of habitat with song structure that might result from variation in acoustic properties with habitat among populations of the same species, among individuals in a population, or among contexts in which an individual uses its songs. At each of these scales, song structures might adapt to habitats. Comparisons among populations of one or a few species might reveal patterns that do not appear in an overall analysis of a diverse avifauna as presented here. However, the scale of the present analysis serves to focus attention on the most prominent patterns in the adaptations of song structure to broad variation in habitats.

Any theories that the properties of songs evolve to minimize distortion during propagation must also consider the possibility that distortion could serve to indicate the distance of a singing bird to a recipient. Several species are known to make use of the distortion of their songs during propagation to judge the distance to a singing conspecific, independent of the overall amplitude of the song (Richards 1981; MacGregor and Falls 1984; MacGregor and Krebs 1984). Experiments have so far not determined whether frequency-dependent attenuation or degradation by reverberation or amplitude fluctuations provide the cues for ranging.

\section{Frequency Characteristics of Song in Relation to Habitat}

Associations between frequencies in songs and habitats have been reported by several previous studies, including both broad surveys of avifaunas (Morton 1975; Ryan and Brenowitz 1985) and comparisons of populations or related species (Jilka and Leisler 1974; Bowman 1979; Hunter and Krebs 1979; Wasserman 1979; Lemon et al. 1981; Shy 1983; Cosens and Falls 1984; Anderson and Conner 1985). In this study, when the confounding influence of body size was controlled, only the maximal frequencies of songs varied consistently with habitat. To see why this pattern might occur, consider the rationale for expecting song frequencies to vary with habitat.

Attenuation of sound in natural environments is affected by three frequencydependent effects: (1) atmospheric absorption, (2) scattering, and (3) ground attenuation as a consequence of propagation in conjunction with waves in the ground and at the surface (Wiley and Richards 1982). The first two produce attenuation that increases monotonically with frequency, at least within ranges of environmental conditions and frequencies applying to birds. However, as scat- 
tering is greater from foliage in forests, the slope of the frequency dependence of attenuation is higher in forests (Morton 1975; Marten and Marler 1977; Marten et al. 1977). In coniferous forests, as a result of the small dimensions of the leaves, attenuation from scattering increases particularly above $8-10 \mathrm{kHz}$ (Marten and Marler 1977). It is also noteworthy that in field conditions the frequency dependence of attenuation has a large amount of irregularity, presumably as a result of standing waves, temporal and spatial variation in conditions, and specific features of scattering in particular environments. Ground effects increase attenuation at low frequencies (generally below $1 \mathrm{kHz}$ or lower), the lower the frequencies the higher above ground (Wiley and Richards 1982; Waser and Brown 1984). In addition, dense vegetation on the ground reduces this effect by absorption of most of the incident sound (Marten and Marler 1977; Cosens and Falls 1984). As their effect is primarily on frequencies below $1 \mathrm{kHz}$, ground effects should have little influence on the songs of North American oscines, which in any case seldom sing advertising songs near the ground (Wiley and Richards 1982). These considerations lead to a prediction that, in all habitats, lower frequencies should attenuate less, except for frequencies below $1 \mathrm{kHz}$ near the ground.

The effective range of a sound, however, depends both on its attenuation and on the background noise. So the acceptable range of frequencies would depend on the relationship of attenuation and background noise (Brenowitz 1982; Waser and Brown 1984). In forests, the steeper frequency dependence of attenuation, in comparison to open habitats, would in general result in a lower upper limit of the band of acceptable frequencies, provided background noise levels did not differ markedly. On the other hand, levels of background noise at high frequencies might differ between habitats and might explain any differences in bands of acceptable frequencies. Ryan and Brenowitz (1985) have provided evidence from limited measurements in Panama that differences in background noise might explain the lower mean frequencies used by birds of forested habitats in comparison to open ones. Similar considerations might apply to arboreal monkeys (Brown and Waser 1984).

These considerations suggest that the three measures of frequencies in this study might depend on habitat and body size in different ways. Dominant frequencies should have the strongest relationship with the size of the sound source and thus with body size. On the other hand, the steeper frequency dependence of attenuation in forests as compared with open habitats suggests that maximal frequencies might show the strongest associations with habitat.

The analyses presented here confirmed these expectations. Of the three measures of frequency, dominant frequency had the strongest association with body mass. On the other hand, maximal frequency was the only one of the three measures that varied convincingly among habitats when body mass was controlled.

It is worth noting, however, that measurements of extreme frequencies, including maximal frequencies, are somewhat problematic. They depend on detecting the limits of traces on spectrograms and thus on the amplitude of the recorded signal in relation to the dynamic range of the spectrum analyzer. All three of these frequency measures also depend on the distance from the source during 
recording and could include systematic biases if the distribution of recording distances varied among habitats.

Previous studies have not explicitly considered these issues in analyzing associations of frequency and habitat. Nevertheless, there is a tendency for maximal frequencies or ranges of frequencies, but not dominant frequencies, to differ among habitats, at least in temperate regions. Several studies have reported higher maximal frequencies or greater ranges of frequencies in open habitats than in forests (Hunter and Krebs 1979; Shy 1983; Anderson and Conner 1985), and one of these studies reported no difference in dominant frequency among habitats (Anderson and Conner 1985). In tropical environments in Panama, however, dominant frequencies varied with habitat (Morton 1975; Ryan and Brenowitz 1985), but possibly as a result of differences in ambient noise levels rather than frequency-dependent attenuation.

The comparison of marshland and grassland species by Cosens and Falls (1984) provides a case for differences in frequencies of birds' songs between major habitats, for which an explanation based on differences in attenuation is particularly plausible. In this case, the absence of a ground effect in marshes, presumably as a result of absorption of sound by dense vegetation covering the surface, reduces attenuation of frequencies below $1 \mathrm{kHz}$ in comparison to grassland. The species that can most effectively make use of this reduced attenuation, however, are relatively large nonpasserine species (see Cosens and Falls 1984). In the present study, oscines in marshes did not use lower dominant or minimal frequencies as compared with open habitats, perhaps because the minimal frequencies in oscines' songs are above the band for significant attenuation by ground effects.

These considerations suggest that the associations of frequencies with major habitats, at least for temperate oscine birds, result from complex and still poorly understood physical constraints on long-range acoustic communication. In fact, this study has revealed pronounced and consistent differences among habitats only for the maximal frequencies in these birds' songs.

\section{Temporal Characteristics of Song in Relation to Habitat}

The expectation of differences among habitats in temporal properties of song rests on much simpler considerations. Sound propagating through scattering environments, like forests, inevitably accumulates reverberation. As scattering is a frequency-dependent process, reverberation also depends on frequency. Measurements suggest that reverberation increases with frequency below $10 \mathrm{kHz}$ but might decrease again at higher frequencies as a result of the increased atmospheric absorption of sound (Richards and Wiley 1980). Reverberation of birds' songs recorded in forests is a conspicuous feature of spectrograms.

Birds can discriminate frequencies nearly as well as mammals in the range of frequencies important for song (Dooling 1982). Because of this frequencyanalyzing property of the avian auditory system, reverberations of sound at any one frequency interfere with subsequent reception of sounds only at nearly the same frequency (specifically, within the masking bandwidth of that frequency). Thus, environments with strong reverberation should favor signals that avoid short repetition periods at a given frequency. The minimal repetition rates mea- 
sured in this study are one index of this feature. Sidebands, particularly sidebands that decrease in intensity monotonically away from the carrier frequency, indicate amplitude modulation of this frequency with a period shorter than the period of the spectrum analysis ( $10 \mathrm{~ms}$ in this study) and thus provide a second index of features degraded by reverberation. The effects of reverberation in this case would smear the sidebands in the frequency domain in a spectrogram. It is probable, however, that avian ears, with temporal resolution near 2-3 ms (Dooling 1982), would hear these sounds as degraded amplitude modulation in the time domain. Buzzes, sounds that appear as band-limited noise in spectrograms, often represent a carrier frequency subject to amplitude modulation with a period close to that of the spectral analysis.

This analysis has shown that almost all oscines of open habitats include very short repetition periods or sidebands in their songs, whereas many birds of forested habitats avoid these features. Nevertheless, many birds of forests do include short repetition periods or sidebands in their songs (figs. 4 and 5). These exceptions might arise as adaptations within individuals' repertoires, within populations, or between populations to differences in microhabitats or social situations. In addition, the retention of short repetition periods in songs of forestinhabiting birds might provide a cue for listeners to judge the distance to the singer, as discussed above.

\section{Implications for Perceptual Adaptations to Different Habitats}

This analysis has provided evidence that the temporal structure of oscine birds' songs in temperate North America is associated in a general way with the habitats in which they live. Could there be corresponding adaptations in the perception of acoustic signals in different habitats?

Some evidence indicates that the auditory systems of oscines are adjusted in a general way to the species-specific features of the frequencies of their songs (Konishi 1970); such evidence is even clearer for arboreal monkeys (Brown and Waser 1984). The possibilities for adaptations to temporal properties of songs have yet to be explored. In mammalian auditory systems, for instance, as a result of both peripheral and central mechanisms, responsiveness to sound recovers during the few milliseconds following reception of an initial sound (Evans 1985). Such damping would prove especially useful in forests to recover the original amplitude pattern from a reverberant signal. Time constants for recovery are known to vary among the few mammals for which this information is available (Evans 1985), but there are no comparisons yet of species inhabiting forested and open habitats. Of course, any such damping would interfere with discrimination of rapid amplitude modulations and would also make it difficult to judge the distance to a source on the basis of levels of reverberation.

In open habitats, however, stronger winds and thermals would create greater temporal variation in the propagation of sound than in forests (Richards and Wiley 1980; Wiley and Richards 1982). As a result of the consequent amplitude fluctuations, sound propagating in open habitats would be subject to variable "dropouts." One might therefore predict that birds of open habitats would have 
evolved neural mechanisms to recognize conspecific song in spite of such dropouts but not to recognize song subjected to reverberation. Birds of forested habitats might have evolved just the opposite adaptations to recognize song after degradation by reverberation but not by dropouts.

\section{ACKNOWLEDGMENTS}

I thank M. Green and R. Godard for many discussions of the ideas presented here. Green in particular helped to develop ideas about perceptual adaptations. A. Grafen kindly offered me a copy of his program for phylogenetic regression; he and M. Ridley contributed suggestions for controlling the effects of phylogeny in analyzing the associations of song and habitat. I also thank P. J. B. Slater, S. Nowicki, O. Larsen, and two reviewers for many helpful suggestions on various stages of the manuscript. This article is a contribution from the Behavioral Research Station in the Mason Farm Biological Reserve, North Carolina Botanical Garden.

\section{LITERATURE CITED}

American Ornithologists' Union. 1983. Check-list of North American birds. 6th ed. American Ornithologists' Union.

Anderson, M. E., and R. N. Conner. 1985. Northern cardinal song in three forest habitats in eastern Texas. Wilson Bulletin 97:436-449.

Bowman, R. I. 1979. Adaptive morphology of song dialects in Darwin's finches. Journal of Ornithology 120:353-389.

Brenowitz, E. A. 1982. The active space of red-winged blackbird song. Journal of Comparative Physiology 147:511-522.

Brown, C. H., and P. M. Waser. 1984. Hearing and communication in blue monkeys (Cercopithecus mitis). Animal Behaviour 32:66-75.

Chappuis, C. 1971. Un exemple de l'influence du milieu sur les émissions vocales des oiseaux: l'évolution des chants en forêt équitoriale. Terre et Vie 25:183-202.

Cornell Laboratory of Ornithology. 1975. Field guide to bird songs. Houghton Mifflin, Boston. Sound cassettes.

Cosens, S. E., and J. B. Falls. 1984. A comparison of sound propagation and song frequency in temperate marsh and grassland habitats. Behavioral Ecology and Sociobiology 15:161-170.

Dooling, R. J. 1982. Auditory perception in birds. Pages 95-130 in D. E. Kroodsma and E. H. Miller, eds. Acoustic communication in birds. Vol. 1. Academic Press, New York.

Dunning, J. B., Jr. 1984. Body weights of 686 species of North American birds. Western Bird Banding Association Monograph 1.

Evans, E. F. 1985. Aspects of the neural coding of time in the mammalian peripheral auditory system relevant to temporal resolution. Pages 74-95 in A. Michelsen, ed. Temporal resolution in auditory systems. Springer, Berlin.

Gish, S. L., and E. S. Morton. 1981. Structural adaptations to local habitat acoustics in Carolina wren songs. Zeitschrift für Tierpsychologie 56:74-84.

Grafen, A. 1989. The phylogenetic regression. Philosophical Transactions of the Royal Society of London B, Biological Sciences 326:119-157.

Handford, P. 1981. Vegetational correlates of variation in the song of Zonotrichia capensis. Behavioral Ecology and Sociobiology 8:203-206.

Healy, M. J. R. 1988. GLIM: an introduction. Clarendon, Oxford. 
Hopkins, C. D., M. Rossetto, and A. Lutjen. 1974. A continuous sound spectrum analyzer for animal sounds. Zeitschrift für Tierpsychologie 34:313-320.

Hunter, M. L., and J. R. Krebs. 1979. Geographical variation in the song of the great tit (Parus major) in relation to ecological factors. Journal of Animal Ecology 48:759-785.

Imhof, T. A. 1962. Alabama birds. University of Alabama Press, Birmingham.

Jilka, A., and B. Leisler. 1974. Die Einpassung dreier Rohrsängerarten (Acrocephalus schoenobaenus, A. scirpaceus, A. arundinaceus) in ihre Lebensräume in bezug auf das Frequenspecktrum ihrer Reviergesänge. Journal für Ornithologie 115:192-212.

Kinsler, L. E., and A. R. Frey. 1962. Fundamentals of acoustics. 2d ed. Wiley, New York.

Konishi, M. 1970. Comparative neurophysiological studies of hearing and vocalizations on song birds. Zeitschrift für vergleichende Physiologie 66:257-272.

Laughlin, S. B., and D. P. Kibbe. 1985. The atlas of breeding birds of Vermont. University Press of New England, Hanover, N.H.

Lemon, R. E., J. Struger, M. J. Lechowicz, and R. F. Norman. 1981. Song features and singing heights of American warblers: maximization or optimization of distance? Journal of the Acoustical Society of America 69:1169-1176.

Marler, P. 1969. Tonal quality of bird sounds. Pages 5-18 in R. A. Hinde, ed. Bird vocalizations. Cambridge University Press, Cambridge.

Marten, K., and P. Marler. 1977. Sound transmission and its significance for animal vocalization. I. Temperate habitats. Behavioral Ecology and Sociobiology 2:271-290.

Marten, K., D. Quine, and P. Marler. 1977. Sound transmission and its significance for animal communication. II. Tropical forest habitats. Behavioral Ecology and Sociobiology 2:291-302.

McCullagh, P., and J. A. Nelder. 1983. Generalized linear models. Chapman \& Hall, London.

McGregor, P. K., and J. B. Falls. 1984. The response of western meadowlarks (Sturnella neglecta) to the playback of undegraded and degraded songs. Canadian Journal of Zoology 62:2125-2128.

McGregor, P. K., and J. R. Krebs. 1984. Sound degradation as a distance cue in great tit (Parus major) song. Behavioral Ecology and Sociobiology 16:49-56.

Morton, E. S. 1975. Ecological sources of selection on avian sounds. American Naturalist 109:17-34.

Murray, B. G., and J. R. Jehl. 1964. Weights of autumn migrants from coastal New Jersey. BirdBanding 35:253-263.

Nottebohm, F. 1975. Continental patterns of song variability in Zonotrichia capensis: some possible ecological correlates. American Naturalist 109:605-624.

Richards, D. G. 1981. Estimation of distance of singing conspecifics by the Carolina wren. Auk 98:127-133.

Richards, D. G., and R. H. Wiley. 1980. Reverberations and amplitude fluctuations in the propagation of sound in a forest: implications for animal communication. American Naturalist 115: 381-399.

Ridley, M. 1983. The explanation of organic diversity. Clarendon, Oxford.

1989. The timing and frequency of mating in insects. Animal Behaviour 37:535-545.

Ryan, M. J., and E. A. Brenowitz. 1985. The role of body size, phylogeny, and ambient noise in the evolution of bird song. American Naturalist 126:87-100.

Salt, W. R., and J. R. Salt. 1976. The birds of Alberta. Hurtig, Edmonton.

Shy, E. 1983. The relation of geographical variation in song to habitat characteristics and body size in North American tanagers (Thraupinae: Piranga). Behavioral Ecology and Sociobiology 12:71-76.

Sorjonen, J. 1986a. Factors affecting the structure of song and the singing behaviour of some northern European passerine birds. Behaviour 98:286-304.

$1986 \mathrm{~b}$. Song structure and singing strategies in the genus Luscinia in different habitats and geographical areas. Behaviour 98:275-285.

Stein, R. C. 1968. Modulation in bird sounds. Auk 85:229-243.

Steward, R. E. 1975. Breeding birds of North Dakota. Tri-college Center for Environmental Studies, Fargo, N.D.

Stewart, E. R., and C. S. Robbins. 1958. Birds of Maryland and the District of Columbia. North American Fauna 62. Department of the Interior, Washington, D.C. 
Wallschläger, D. 1980. Correlation of song frequency and body weight in passerine birds. Experientia $36: 412$.

Waser, P. M., and C. H. Brown. 1984. Is there a "sound window" for primate communication? Behavioral Ecology and Sociobiology 15:73-76.

Wasserman, F. E. 1979. The relationship between habitat and song in the white-throated sparrow. Condor 81:424-426.

Wiley, R. H., and D. G. Richards. 1978. Physical constraints on acoustic communication in the atmosphere: implications for the evolution of animal vocalizations. Behavioral Ecology and Sociobiology 3:69-94.

1982. Adaptations for acoustic communication in birds: sound transmission and signal detection. Pages 131-181 in D. E. Kroodsma and E. H. Miller, eds. Acoustic communication in birds. Vol. 1. Academic Press, New York. 